\title{
Meta-Analysis of Regression Coefficients for the Relationship Between Fusarium Head Blight and Deoxynivalenol Content of Wheat
}

\author{
P. A. Paul, P. E. Lipps, and L. V. Madden
}

Department of Plant Pathology, The Ohio State University, Ohio Agricultural Research and Development Center, Wooster 44691. Accepted for publication 23 March 2006.

\section{ABSTRACT}

Paul, P. A., Lipps, P. E., and Madden, L. V. 2006. Meta-analysis of regression coefficients for the relationship between Fusarium head blight and deoxynivalenol content of wheat. Phytopathology 96:951-961.

A total of 126 field studies reporting deoxynivalenol (DON; ppm) content of harvested wheat grain and Fusarium head blight index (IND; field or plot-level disease severity) were analyzed to determine the overall mean regression slope and intercept for the relationship between DON and IND, and the influence of study-specific variables on the slope and intercept. A separate linear regression analysis was performed to determine the slope and intercept for each study followed by a meta-analysis of the regression coefficients from all studies. Between-study variances were significantly $(P<0.05)$ greater than 0 , indicating substantial variation in the relationship between the variables. Regression slopes and intercepts were between -0.27 and $1.48 \mathrm{ppm}$ per unit IND and -10.55 to $32.75 \mathrm{ppm}$, respectively. The overall mean regression slope and intercept, $0.22 \mathrm{ppm}$ per unit IND and $2.94 \mathrm{ppm}$, respectively, were significantly different from zero $(P<0.001)$, and the width of the $95 \%$ confidence interval was $0.07 \mathrm{ppm}$ per unit IND for slope and $1.44 \mathrm{ppm}$ for intercept. Both slope and intercept were significantly affected by wheat type $(P<$ 0.05 ); the overall mean intercept was significantly higher in studies conducted using winter wheat cultivars than in studies conducted using spring wheat cultivars, whereas the overall mean slope was significantly higher in studies conducted using spring wheat cultivars than in winter wheat cultivars. Study location had a significant effect on the intercept $(P<0.05)$, with studies from U.S. winter wheat-growing region having the highest overall mean intercept followed by studies from Canadian wheat-growing regions and U.S. spring wheat-growing regions. The study-wide magnitude of DON and IND had significant effects on one or both of the regression coefficients, resulting in considerable reduction in between-study variances. This indicates that, at least indirectly, environment affected the relationship between DON and IND.

Additional keywords: bivariate mixed-effects models, bivariate randomeffects models, effect size, Fusarium graminearum, Gibberella zeae, maximum likelihood, wheat scab.
Fusarium head blight of wheat (Triticum aestivum L.), caused primarily by Fusarium graminearum Schwabe (teleomorph: Gibberella zeae), has become a yield-limiting factor in many wheat and barley production regions of the world $(1,25,29)$. Both direct and indirect losses may result from Fusarium head blight infection $(25,44)$. Direct losses are due primarily to reduced production per unit area. Severely infected spikes yield shriveled, small, light-weight kernels, leading to low test weights. In addition, cultivation of infected seeds leads to poor seed germination and seedling blight, and consequently, low plant populations per unit area. Estimated yield losses due to the Fusarium head blight epidemic of 1993 in North Dakota, Minnesota, South Dakota, and Manitoba, Canada totaled 4.78 million metric tons (25). Between 1998 and 2000, total estimated losses due to Fusarium head blight across all wheat classes (hard red spring, soft red winter, and durum wheat) and all major U.S. wheat-growing regions were estimated at 1.30 million metric tons (27). In Ohio, the Fusarium head blight epidemic of 1996 led to an estimated $40 \%$ reduction in wheat yield (20).

In addition to direct yield loss, wheat growers may incur further loss as a result of poor grain quality. Grain harvested from affected fields may have a high percentage of Fusarium-damaged kernels and a high concentration of deoxynivalenol (DON), a mycotoxin produced by $F$. graminearum. Even when kernels appear wholesome, DON levels may exceed predetermined thresholds

Corresponding author: L. V. Madden; E-mail address: madden.1 @ osu.edu

DOI: 10.1094/PHYTO-96-0951

(C) 2006 The American Phytopathological Society for commercialization and consumption $(32,44)$. This information is often gathered only at the time of marketing when the grain is either rejected or priced down because of unacceptably high levels of DON. By that time, growers would have already incurred expenses associated with harvesting and marketing of their grain. Although there is a positive association between Fusarium head blight intensity and DON content of harvested grain (31), low levels (or absence) of visible Fusarium head blight damage does not necessarily translate into proportionally low levels of DON-contaminated grain (11).

A meta-analysis of the relationship between visual estimates of Fusarium head blight intensity and DON accumulation in harvested grain revealed that, overall, there was a significant positive relationship between DON and three measures of Fusarium head blight intensity in the field, incidence (proportion of diseased spikes per sample), diseased head severity (average percentage of diseased spikelets per diseased spike), and "index" (also referred to as plot or field severity, that is, the average percentage of diseased spikelets per spike) (31). However, the highest correlations were between DON and index. The overall mean correlation coefficient for the relationship between index and DON was 0.62. Approximately $70 \%$ of the 158 studies analyzed had correlation coefficients $>0.50$. Whereas the correlation coefficient gives an excellent measure of the strength of the linear association between variables, and is considered by Hunter and Schmidt (16) as the preferred statistic to determine if there is a significant relationship between two variables in a meta-analysis, it is essentially only a measure of the spread of points about the regression line and a measure of the change in standardized DON values with standardized index values. Little inference can be drawn about the change in DON levels with changing Fusarium head blight index 
(IND), or mean DON levels at a given value of index, that is, the slope and intercept of the best-fitted regression line relating DON to disease index. It is possible for two separate studies of the relationship between a pair of variables to yield similar correlation coefficients but different regression slopes and intercepts or similar regression parameters but different correlation coefficients.

Direct quantification of DON is time-consuming and expensive. Efforts are currently being made to estimate DON levels using empirical, weather-based models (14). Other forecasting approaches are based on predicting the risk of high disease index (often with disease index being used as an implicit indicator of DON levels) $(4,5,24)$. These modeling efforts could benefit from a better understanding of the relationship between Fusarium head blight intensity and DON content of grain. Empirical DON prediction models could provide growers with early information regarding DON levels in harvested grain. Using such a tool, growers could make more informed decisions regarding harvesting and marketing of their grain.

The objectives of this study were to conduct meta-analyses to determine (i) whether there is a straight-line relationship between DON at harvest and disease index at Feekes growth stage 11.2 (soft dough; 19); (ii) the overall mean rate of change in DON content with change in disease index across multiple studies, and the mean DON content at a given index level (measured by the regression line intercept); (iii) whether the overall regression parameter estimates were influenced by wheat-growing region (U.S. spring, U.S. winter, and Canadian wheat-growing regions), wheat type (spring versus winter wheat) and study type (fungicide trials versus genotype trials); and (iv) whether critical levels of DON could be reliably estimated from known levels of IND and the overall mean index corresponding to each critical DON level.

\section{MATERIALS AND METHODS}

Database for meta-analysis. Data from 126 studies consisting mainly of Fusarium head blight fungicide trials and diseasescreening nurseries evaluations were compiled for this analysis. These studies were acquired from the database of the U.S. Wheat and Barley Scab Initiative (http://www.scabusa.org) and from collaborators (D. Hershman, C. Sneller, M. McMullen, and G. Milus) and represent a subset of the total number of studies used in a previous meta-analysis (31). Studies with missing DON or disease index data, that is, those only reporting correlations, were omitted from the original list of studies. A detailed description of the data set and the criteria used for selecting the studies were provided previously (31). In brief, these studies were conducted between 1998 and 2004 at multiple locations across the wheatproducing regions of the United States and Canada. Sixty-six studies were from U.S. winter wheat-producing areas (Mid-Atlantic, southern, and Midwestern regions not including Iowa, Minnesota, and the Dakotas), 45 from U.S. spring wheat-producing areas (Iowa, Minnesota, and the Dakotas), and the remaining 15 from Canada (13 from Ontario and two from Manitoba).

Both the fungicide trials and disease-screening nurseries were conducted according to standard protocols established by collaborating researchers through the U.S. Wheat and Barley Scab Initiative. The genotypes evaluated in each disease-screening nursery included breeding lines from regional wheat-breeding programs and check cultivars that ranged from highly susceptible to moderately resistant to Fusarium head blight. Between 22 and 70 genotypes were evaluated in each nursery. Each fungicide trial consisted of between 6 to 14 treatments (products, application rates, and application times) applied to a susceptible cultivar, plus an untreated control.

Artificial inoculations, either through the atomization of a spore suspension of $F$. graminearum onto wheat spikes at flowering or the spread of $F$. graminearum-infested maize kernels throughout the plots, were performed in most of the studies. In some cases, plots were planted into maize or wheat residue. Subsequent to inoculation, plots in some trials were mist-irrigated to enhance disease development. IND (average percentage of diseased spikelets per spike) was either estimated visually at approximately Feekes growth stage 11.2 or calculated from visual estimates of disease incidence (INC, proportion of diseased spikes in a sample) and diseased-head severity (DHS, average percentage of diseased spikelets per diseased spike) as IND $=$ DHS $\times$ INC (38). DON was quantified (in parts per million) after harvest as the amount of DON per unit weight of a bulked sample of ground kernels as described by Hart et al. (10).

Meta-analysis of regression slopes and intercepts. Within each study, each pair of DON and disease index values (generally averaged across three or four replicate plots) was considered an observation for data analysis. Data from individual replicates were unavailable for most studies. Hence, the total number of observations $(N)$ for each study corresponded to the total number of genotypes ( 22 to 70 ; for genotype trials) or the total number of treatments plus the untreated control ( 7 to 15 ; for fungicide trials) in the study. A preliminary meta-analysis was performed to determine if there was a linear relationship (or an overall curvature to the relationship) between DON and IND. Correlations between DON and various power transformations of IND $\left(\mathrm{IND}^{1 / 3}, \mathrm{IND}^{1 / 2}\right.$, $\mathrm{IND}, \mathrm{IND}^{2}$, and $\mathrm{IND}^{3}$ ) were calculated for each study. Then, a separate random-effects meta-analysis was conducted based on the Fisher $z_{r}$ transformation for each of these correlations; mean correlation and standard error were determined using methods identical to that described in Paul et al. (31). A concave relationship between DON and IND would result in higher correlation when IND was raised to a fractional power (e.g., 1/2); a convex relationship would result in a higher correlation when IND was raised to a power $>1$.

A separate ordinary least squares regression analysis was performed for each study using PROC REG of SAS (SAS Institute, Cary, NC). The linear model is written as

$$
\mathrm{DON}_{j}=\alpha+\beta \mathrm{IND}_{j}+\varepsilon_{j}
$$

in which $j$ is the index for the individual DON/IND values within each study $(j=1, \ldots, N), \alpha$ is the expected DON concentration when IND $=0$ (the "intercept"), $\beta$ is the slope, the expected change in DON concentration with unit increase in disease index, and $\varepsilon$ is the residual or error term (the difference between observed DON and that specified by the deterministic part of the model). The estimates of $\alpha$ and $\beta$ are written as $a$ and $b$, respectively. In addition to the parameter estimates, the coefficient of determination $\left(r^{2}\right)$, standard deviation about the regression line $(s)$, and standard errors of the estimated parameters $\left(s_{a}\right.$ and $\left.s_{b}\right)$ were calculated. The square root of $r^{2}$ is the correlation coefficient considered in Paul et al. (31). Appropriateness of the linear model was evaluated through the use of residuals, studentized residuals, and Cook's distances (34), the latter being an indication of the effect of each observation (within a study) on $a$ and $b$. The estimated regression coefficients and their corresponding standard errors were then combined with summary statistics (mean $[\bar{x}]$, maximum [Max], minimum [Min], and $N$ for DON and disease index) and categorical moderator variables (study type, wheat type, and study location) and subjected to metaanalyses.

Estimated regression slopes $(b)$ and intercepts $(a)$ were the effect sizes of interest for each study. A bivariate random-effects meta-analysis was performed to estimate the overall mean effect sizes (mean slope $[\bar{b}]$ and intercept $[\bar{a}]$ ) and to determine the variability in effect sizes among studies. Random-effects models were fitted in PROC MIXED of SAS as described by Normand (28), Stijnen (39), and Sheu and Suzuki (36) and subsequently expanded for the bivariate setting as described by van Houwelingen et al. (40). In PROC MIXED, meta-analysis is implemented with- 
in the framework of linear mixed models (22). The bivariate metaanalytical model can be written in matrix format as

$$
\left(\begin{array}{l}
a \\
b
\end{array}\right)_{i} \sim N\left(\mu, \Sigma+\mathrm{C}_{i}\right)
$$

where $\sim N(\bullet)$ indicates a (bivariate) normal distribution, $\mu$ is the vector of expected values of intercept and slope (i.e., the effect size vector, consisting of the unknown intercept, $\alpha$, and slope, $\beta$ ), $\Sigma$ is the matrix of between-study variances and covariance of the intercept and slope, and $C_{i}$ is the matrix of within-study (sampling) variances for the intercept and slope for the $i$ th study. The vectors and matrices can be written as

$$
\mu=\left(\begin{array}{l}
\alpha \\
\beta
\end{array}\right), \Sigma=\left(\begin{array}{cc}
\sigma_{a}^{2} & \sigma_{a b} \\
\sigma_{a b} & \sigma_{b}^{2}
\end{array}\right), C_{i}=\left(\begin{array}{cc}
s_{a}^{2} & 0 \\
0 & s_{b}^{2}
\end{array}\right)_{i}
$$

in which $s_{a}^{2}$ and $s_{b}^{2}$ are the squares of the standard errors of the intercept and slope for each study, $\sigma_{a}^{2}$ and $\sigma_{b}^{2}$ are the betweenstudy variances of the intercept and slope, and $\sigma_{a b}$ is the between-study covariance of the estimated intercept and slope. In random-effects meta-analysis, it is assumed that the $C_{i}$ matrix is fixed for each study (determined from the individual studies before the meta-analysis), and $\mu$ and $\Sigma$ are estimated from the data. The bivariate model was fitted with PROC MIXED by using maximum likelihood to estimate the $\Sigma$ matrix elements and generalized least squares to estimate elements of $\mu$, in a manner equivalent to the protocol shown on pages 611-612 of van Houwelingen et al. (40). The estimated effect-size vector can be written as

$$
\hat{\mu}=\mathrm{E}=\left(\begin{array}{l}
\bar{a} \\
\bar{b}
\end{array}\right)
$$

As with univariate meta-analysis, in determining $\hat{\mu}$, studies are weighted in inverse proportion to the sampling variances $\left(s_{a}^{2}\right.$ and $\left.s_{b}^{2}\right)$. Significance of effect size was determined with a standard normal test statistic $(9,13,21)$. Standard errors of $\bar{a}$ and $\bar{b}$ were determined by PROC MIXED from $\Sigma$ and $C_{i}$ based on mixedmodel theory (22).

Influence of study characteristics on the effect sizes. Since the data used herein were from different sources and different types of studies, the bivariate meta-analytic approach was expanded to determine the influence of certain specific study characteristics (moderator variables) on the effect sizes. Categorical moderator variables were used to classify each study based on the type of study (fungicide evaluation versus genotype screening), the type of wheat used in the study (spring wheat versus winter wheat), and study location (traditional U.S. winter wheat-, U.S. spring wheat-, and Canadian wheat-producing areas). In addition, mean, maximum, and the difference between mean and maximum ("half-range"; Rng) were calculated for DON and IND for each study and used as continuous moderator variables. As discussed in Paul et al. (31), the mean and maxima can be considered indirect measures of environmental favorability for disease development or DON production. The mean and maxima could also reflect degree of pathogen aggressiveness, although investigators typically used an aggressive isolate or group of isolates of the pathogen in the individual studies. The Rng indicates the extent to which IND and DON varied within individual studies, which could affect regression and correlation results (17). A separate bivariate meta-analysis was performed for each moderator variable to determine whether the variable significantly affected the effect sizes (i.e., the vector of $a$ and $b$ in equation 2 ) and to estimate separate mean effect sizes for each level of categorical moderate variable. All analyses of moderator variable effects were also done with PROC MIXED. The model is of the same format as equation 2 , but with $\mu$ decomposed into terms for categorical or continuous variables. The general expression for $\mu$ can be written as $\mu=X \gamma$, in which $X$ is the matrix of the continuous variables or indicator variables for the categorical variables (e.g., study type), and $\gamma$ is the matrix of unknown coefficients for the effect of $X$ on the effect size. Linear contrasts were used to estimate mean effect sizes (or differences of mean effect sizes) for levels of the categorical variables. Significance of continuous and categorical variances on effect size was determined using chi-square tests $(9,13,21)$ based on the linear contrasts. All moderator variables were considered fixed effects in the models; thus, the bivariate meta-analytical models with moderator variables are considered mixed-effects models.

Linear mixed models with or without correlated data are robust to moderate departures from normality of the random effects (42). However, to evaluate the reasonableness of the normality assumption and the general appropriateness of the bivariate model, plots of the studentized residuals were obtained using the residual option in PROC MIXED.

\section{RESULTS}

Meta-analysis of correlation coefficients and regression analysis. A preliminary meta-analysis of Fisher-transformed correlation coefficients $\left(Z_{r}\right)$ was performed to ascertain whether there was an overall curvature to the relationship between DON and IND. The overall mean transformed correlation coefficients $\left(\bar{Z}_{r}\right)$ for the relationship between DON and various power transformations of IND were similar to, but lower than, $\bar{Z}_{r}$ for the relationship between DON and untransformed IND. Values of $\bar{Z}_{r}$ were $0.64,0.65,0.67,0.64$, and 0.60 for $\mathrm{IND}^{1 / 3}, \mathrm{IND}^{1 / 2}$, IND, $\mathrm{IND}^{2}$, and $\mathrm{IND}^{3}$, respectively. For all relationships, $\bar{Z}_{r}$ was significantly different from zero based on the standard normal test $(P<0.001)$. Based on the 126 studies, the mean correlation between DON and untransformed IND, determined from the backtransformation of $\bar{Z}_{r}$, was 0.58 , with a $95 \%$ confidence interval of 0.53 to 0.63 . This mean correlation was slightly lower than the mean value for the 158 studies in Paul et al. (31). Only the correlations, but not the original data, were available for 32 studies in Paul et al. (31) which could not be considered here. Residual plots were also determined for each of the individual studies based on a linear model, and a random pattern was found for most studies (P. A. Paul and L. V. Madden, unpublished data). Since there was no overall evidence of curvature to the relationship between DON and IND, regression analyses were based on the untransformed IND. Furthermore, based on Cook's distances (34), only $1 \%$ of the observations had a large influence on the estimated intercepts and slopes. These mostly corresponded to the no-fungicide treatment in the fungicide studies and the most susceptible genotype in the genotype studies. However, half of these high impact observations had positive residuals and half had negative residuals. Thus, even the most influential observations had no consistent effect on the observed relationship between DON and IND, demonstrating the robustness of the linear model utilized (equation 1).

Based on the values of the adjusted coefficient of determination, for more than $60 \%$ of the 126 studies, $20 \%$ or more of the variation in DON content was explained by variation in IND. In $31 \%$ of the studies, $>50 \%$ of the variation in DON was explained by IND. Regression slopes and intercepts varied among studies, ranging from -0.27 to $1.48 \mathrm{ppm}$ per unit IND for the slope $(b)$ and -10.55 to $32.75 \mathrm{ppm}$ for the intercept (a) (Fig. 1A and B). Ninety percent of the studies had slopes greater than zero, with $84 \%$ being between 0 and $0.6 \mathrm{ppm}$ per unit IND. Seventy-seven percent of the intercepts were between 0 and 13 ppm (Fig. 1). Graphically, there was no evidence of a strong correlation between the estimates of the slope and intercept (Fig. 1C). Moreover, no distinct pattern was observed in the relationship between the number of observations in each study and the estimated regression coefficients (data not shown), suggesting that sample size had little effect on the regression slope and intercept. 
Meta-analysis of regression coefficients. Based on the $Z$ statistic from the bivariate meta-analysis, both mean effect sizes were significantly different from zero $(P<0.001$; Table 1$)$. The estimated mean slope was $0.22 \mathrm{ppm}$ per unit IND, with a standard error of 0.017 . The estimated overall mean intercept was $2.94 \mathrm{ppm}$, with a standard error of 0.367 . The widths of the $95 \%$ confidence intervals around the mean slope and intercept were $0.068 \mathrm{ppm}$ per unit IND and $1.438 \mathrm{ppm}$, respectively. Thus, based on the mean regression parameters from the 126 studies analyzed, for every unit increase in IND there was on average a $0.22 \mathrm{ppm}$ increase in
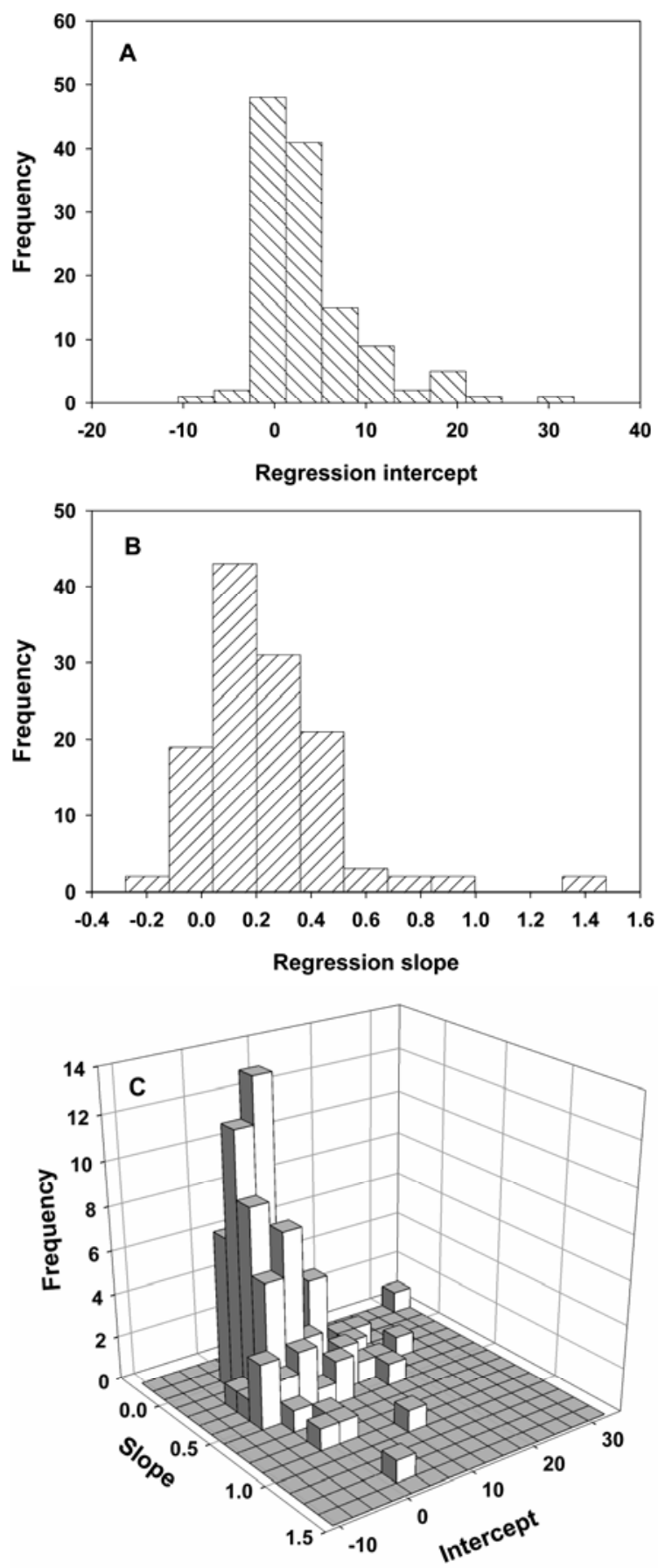

Fig. 1. Empirical distribution of $\mathbf{A}$, estimated regression intercepts and $\mathbf{B}$, slopes, and $\mathbf{C}$, bivariate histogram of regression slopes and intercepts, from 126 studies of the relationships between deoxynivalenol accumulation in harvested wheat grain (ppm) and Fusarium head blight index (IND) (mean percentage of diseased spikelets per spike). Two outliers, one for slope (4.98 ppm/\% IND) and one for intercept (47.98 ppm), were removed before plotting the graphs.
DON; furthermore, when there was no visual symptoms of Fusarium head blight (IND $=0$ ), the overall mean DON level was estimated to be $2.94 \mathrm{ppm}$.

The estimated between-study variances were significantly greater than zero for both the slope and the intercept (Table 1), based on a standard normal test. The estimated between-study covariance was -0.146 ( $\mathrm{se}=0.074)$, which was also significant $(P<$ $0.05)$. With this covariance, the between-study correlation between $\bar{a}$ and $\bar{b}$ was 0.22 . Results of the standard normal tests were confirmed with the likelihood ratio test for random effects, as described by Littell et al. (22). The nonzero variances are justification for the random-effects (rather than fixed-effects) metaanalysis.

Moderator variables. The influence of moderator variables on the overall mean effect sizes (regression coefficients) varied from no detectable effect to highly significant effect (Tables 2 and 3). Neither slope nor intercept were significantly affected by study type. Genotype evaluation studies had higher overall mean slope and intercept than fungicide trials, but the difference in mean slope and intercept between the two types of study was not statistically significant $(P>0.05$; Table 2$)$. Study location had a significant effect on the vector of mean effect sizes. In particular, location affected intercept, with studies from U.S. winter wheatgrowing region having the highest overall mean intercept, followed by studies from Canadian wheat-growing regions and U.S. spring wheat-growing regions. The overall mean slope was only marginally affected by study location $(P \approx 0.07)$. The combined results for slope and intercept indicated that for any fixed value of IND below 25\%, the highest expected DON was for U.S. winter wheat locations. Above 25\% IND, the highest expected DON was for U.S. spring wheat locations, the lowest expected DON was for Canada, with intermediate values for U.S. winter wheat locations.

Slope and intercept were strongly influenced by wheat type. The overall mean intercept was significantly higher in studies using winter wheat cultivars than in studies using spring wheat cultivars (Table 2). The opposite trend was observed for the slope, with studies using spring wheat cultivars having significantly higher overall mean slope than those using winter wheat cultivars. Because of the combined results for intercept and slope, the ranking of expected DON values for wheat type depended on the IND value. At fixed values of IND $<23 \%$, expected DON was higher in studies with winter wheat cultivars than in those with spring wheat cultivars. Above $23 \%$ IND, the trend was reversed, with expected DON being higher in studies with spring wheat cultivars than in those with winter wheat cultivars.

Relative to the model fitted without moderator variables, most of the models fitted after the inclusion of moderator variables had only marginally smaller between-study variances for both the intercept and slope (Table 4). Categorical moderator variables explained between 4.7 and $11.1 \%$ of the between-study variance for regression slopes and between 0 and $6.4 \%$ of the between-study variance for regression intercepts (Table 4). Thus, even when a moderator variable had a significant effect, there remained considerable variability among studies.

Among the continuous moderator variables, mean DON ( $\left.\bar{X}_{\mathrm{DON}}\right)$, mean IND $\left(\bar{X}_{\mathrm{IND}}\right)$, maximum DON $\left(\operatorname{Max}_{\mathrm{DON}}\right)$, and the difference between maximum and mean DON (Rng $g_{\mathrm{DON}}$; roughly half the data range) had the strongest influences on the overall mean effect sizes. The bivariate meta-analytical regression models for the relationship between each of these moderators and the joint effect sizes were statistically significant $(P<0.05$; Table 3$)$ based on the chi-square test. Based on tests of contrasts, however, the continuous variables may have affected just the slope $(b)$ or intercept (a) (Table 3). Increasing $\bar{X}_{\mathrm{IND}}, \bar{X}_{\mathrm{DON}}, \operatorname{Max}_{\mathrm{DON}}$, and $R n g_{\mathrm{DON}}$ resulted in increasing intercept.

Continuous moderator variables explained between 0.5 and $61.5 \%$ and 0 and $45.2 \%$ of the between-study variances for intercepts and slopes, respectively (Table 4). The inclusion of DON- 
related moderator variables resulted in the greatest reduction of the between-study variances associated with both slope and intercept (Table 4). By including $\bar{X}_{\text {DON }}$, the variance of the intercept was reduced by $61.5 \%$ and the variance of the slope was reduced by $35.5 \%$. The inclusion of $\operatorname{Max}_{\mathrm{DON}}$ or the difference between $\operatorname{Max}_{\mathrm{DON}}$ and $\bar{X}_{\text {DON }}$ reduced the between-study variance of the slope by $45.2 \%$.

Estimating expected DON from IND. Using the estimated parameters from the meta-analyses ( $a$ and $b$ ) (Tables 1 and 2), regression lines (predicted mean DON values for a range of IND values) and their corresponding 95\% confidence intervals were plotted for all studies and for studies grouped on the basis of wheat type, wheat-growing region, and study type (Fig. 2). The confidence interval was given as $\mathrm{DÔN} \pm 1.96 \sqrt{V(\mathrm{DOON})}$, where DÔN $=\bar{a}+\bar{b}$ IND, and $V$ (DÔN) is the variance of the estimated expected DON, calculated as $s e^{2}(\bar{a})+\mathrm{IND}^{2} \cdot s e^{2}(\bar{b})+$ $2 \cdot \operatorname{IND} \cdot \operatorname{Cov}(\bar{a}, \bar{b})$ (based on large-sample statistical theory for the variance of a linear combination of two correlated random variables $[a$ and $b]) . \operatorname{Cov}(\bullet)$ is the estimated covariance of the two effect sizes, and $\operatorname{se}(\bullet)$ is the estimated standard error given in Table 2 for each effect size. Confidence intervals around a regression line give a measure of the likely region where the expected DON

TABLE 1. Mean regression coefficients (effect sizes) and their corresponding statistics from a bivariate random-effects meta-analysis of the relationship between Fusarium head blight index (IND) and deoxynivalenol (DON; ppm) content of harvested wheat grain

\begin{tabular}{|c|c|c|c|c|c|c|c|c|c|c|}
\hline \multirow[b]{2}{*}{ Coefficients $^{\mathrm{a}}$} & \multicolumn{6}{|c|}{ Effect size $(E)^{\mathrm{b}}$} & \multicolumn{4}{|c|}{ Between-study variance ${ }^{c}$} \\
\hline & $\bar{E}$ & $\operatorname{se}(\bar{E})$ & $\mathrm{CI}_{L}$ & $\mathrm{CI}_{U}$ & $Z$ & $P$ value & $\hat{\sigma}^{2}$ & $\operatorname{se}\left(\hat{\sigma}^{2}\right)$ & $Z$ & $P$ value \\
\hline Slope $(b)$ & 0.22 & 0.017 & 0.18 & 0.25 & 12.51 & $<0.001$ & 0.03 & 0.01 & 5.85 & $<0.001$ \\
\hline Intercept $(a)$ & 2.94 & 0.366 & 2.22 & 3.65 & 8.01 & $<0.001$ & 14.08 & 2.41 & 5.85 & $<0.001$ \\
\hline
\end{tabular}

${ }^{a}$ Slope and intercept from ordinary least squares regression analysis of the relationship between IND (mean percentage of diseased spikelets per spike) and DON.

b $\bar{E}=$ overall mean effect sizes (slope and intercept); $\operatorname{se}(\bar{E})=$ standard error of $\bar{E}$; lower $\left(\mathrm{CI}_{L}\right)$ and upper $\left(\mathrm{CI}_{U}\right)$ limits of the $95 \%$ confidence interval $(\mathrm{CI})$ around $\bar{E} ; Z$ (standard normal) statistic $P=$ probability value (significance level) from bivariate random-effects meta-analysis of linear regression coefficients from 126 studies.

${ }^{c} \hat{\sigma}^{2}=$ estimated between-study variance; se $\left(\hat{\sigma}^{2}\right)=$ standard error of $\hat{\sigma}^{2}$; and $Z$ (standard normal) statistic and $P=$ probability value (significance level) for testing of the equality of the estimated random effects variance to zero.

TABLE 2. Statistics from bivariate random-effects meta-analysis of the influence of study type, study location, and wheat type on the overall mean regression slope and intercept for relationship between Fusarium head blight index (IND; mean percentage of diseased spikelets per spike) and deoxynivalenol (DON; ppm) content of harvested wheat grain

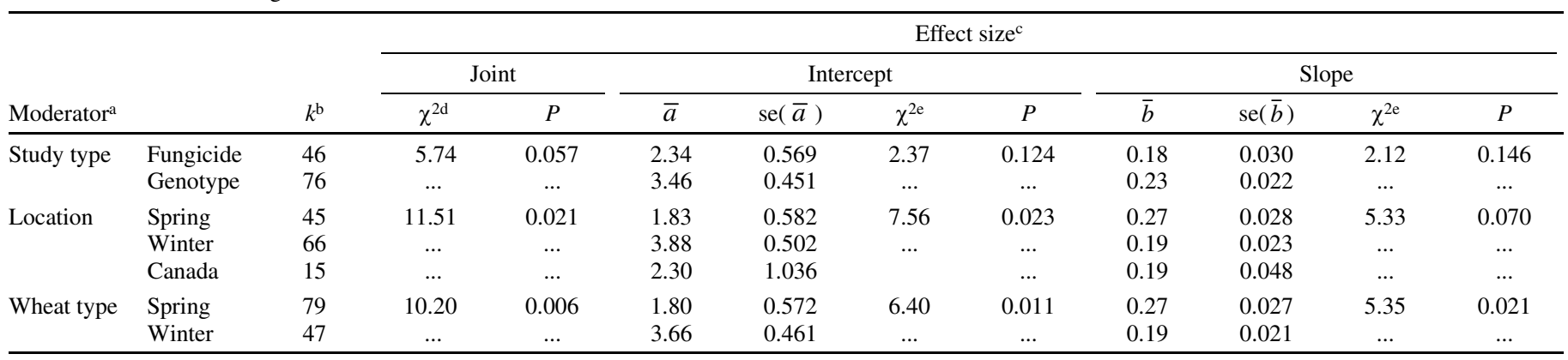

a Study type = data from fungicide trials and genotype evaluation studies; location = data from studies conducted in U.S. winter wheat-, U.S. spring wheat-, and Canadian wheat-growing areas; and wheat type $=$ data from studies conducted using winter wheat and spring wheat cultivars.

b Total number of studies in each category of each moderator variable.

c Joint regression coefficients, overall mean intercept, $\bar{a}$; overall mean slope, $\bar{b}$; standard error of $\bar{a}$, se $(\bar{a})$; standard error of $\bar{b}$, se $(\bar{b})$; and probability value $(P)$ (significance level) for testing the effects of moderator variables on joint and individual regression coefficients.

${ }^{d}$ Chi-square statistic for the effect of the moderator variables on the joint effect size ( $\bar{a}$ and $\bar{b}$ vectors). Degrees of freedom were 2,4 , and 2 for study type, location, and wheat type, respectively.

e Chi-square statistic for the effect of the moderator variables on the intercept $(\bar{a})$ and slope $(\bar{b})$. Degrees of freedom were 1,2 , and 1 for study type, location, and wheat type, respectively.

TABLE 3. Statistics from bivariate random-effects meta-analysis of the influence of mean, maximum, and half-range for deoxynivalenol (DON; ppm) concentration and Fusarium head blight index (IND; mean percentage of diseased spikelets per spike) on the overall mean regression slope and intercept for relationship between IND and DON content of harvested wheat grain

\begin{tabular}{|c|c|c|c|c|c|c|c|c|c|c|c|}
\hline \multirow[b]{3}{*}{ Moderator $^{\mathrm{a}}$} & \multicolumn{11}{|c|}{ Effect size $^{b}$} \\
\hline & \multirow{2}{*}{$\begin{array}{c}\text { Joint } \\
\chi^{2}(P)^{\mathrm{c}}\end{array}$} & \multicolumn{5}{|c|}{ Intercept $(a)$} & \multicolumn{5}{|c|}{ Slope $(b)$} \\
\hline & & $\hat{\gamma}_{0}$ & $\operatorname{se}\left(\hat{\gamma}_{0}\right)$ & $\hat{\gamma}_{1}$ & $\operatorname{se}\left(\hat{\gamma}_{1}\right)$ & $\chi^{2}(P)^{\mathrm{d}}$ & $\hat{\gamma}_{0}$ & $\operatorname{se}\left(\hat{\gamma}_{0}\right)$ & $\hat{\gamma}_{1}$ & $\operatorname{se}\left(\hat{\gamma}_{1}\right)$ & $\chi^{2}(P)^{\mathrm{d}}$ \\
\hline $\bar{X}_{\mathrm{DON}}$ & $289.12(<0.001)$ & -0.20 & 0.343 & 0.497 & 0.041 & $144.73(<0.001)$ & 0.12 & 0.020 & 0.010 & 0.002 & $26.15(<0.001)$ \\
\hline $\operatorname{Max}_{\mathrm{IND}}$ & $3.45(0.179)$ & 1.96 & 0.640 & 0.024 & 0.013 & $3.41(0.065)$ & 0.23 & 0.034 & -0.0003 & 0.0006 & $0.25(0.615)$ \\
\hline $\operatorname{Max}_{\mathrm{DON}}$ & $110.50(<0.001)$ & 1.26 & 0.487 & 0.106 & 0.022 & $23.86(<0.001)$ & 0.11 & 0.019 & 0.005 & 0.0008 & $45.18(<0.001)$ \\
\hline$R n g_{\mathrm{IND}}$ & $1.05(0.591)$ & 2.48 & 0.592 & 0.021 & 0.021 & $0.07(0.326)$ & 0.21 & 0.030 & 0.0001 & 0.001 & $0.01(0.917)$ \\
\hline
\end{tabular}

a Continuous moderator variables: $\bar{X}=$ arithmetic mean, Max = maximum, and Rng = difference between maximum and mean (half-range) for IND and DON.

${ }^{\mathrm{b}}$ Regression intercept and slope from 126 studies; slope $\left(\hat{\gamma}_{1}\right)$, intercepts $\left(\hat{\gamma}_{0}\right)$ and their corresponding standard errors (se), and chi-square statistic $\left(\chi^{2}\right)$ and probability value $(P)$ from meta-analysis of the effects of continuous moderator variables on the overall mean slope $(b)$ and intercept $(a)$.

${ }^{\mathrm{c}}$ Chi-square statistic for the effect of the moderator variables on the joint effect size ( $\bar{a}$ and $\bar{b}$ vectors). Degrees of freedom $=2$.

${ }^{\mathrm{d}}$ Chi-square statistic for the effect of the moderator variables on the intercept $(\bar{a})$ and slope $(\bar{b})$. Degrees of freedom $=1$. 
lies and show how precisely the regression function was estimated (17). The width of the interval reflects the within-study and between-study variances and covariance, and number of studies in a group, all of which determine $\operatorname{se}(\bullet)$ and $\operatorname{Cov}(\bullet)$. From the plots in Figure 2, the general trends in the relationships between DON and IND were similar for all subgroups of studies. However, the width of the $95 \%$ confidence intervals varied from one subgroup to another. Based on the width of the confidence intervals, the regression functions were estimated more precisely for studies grouped according to study type (Fig. 2B and C) and wheat type (Fig. $2 \mathrm{G}$ and $\mathrm{H}$ ) than for studies grouped on the bases of wheatgrowing region (Fig. 2D, E, and F). The wider interval for Canadian studies clearly reflected the relatively small number of studies considered. Irrespective of the subgroups used, the level of DON was estimated more precisely at lower IND values than at higher values. The narrowest widths of the confidence intervals were around 5\% IND. The increasing width of the intervals with increasing IND was due to the relatively small number of observations within studies with very large IND values (that is, large values that were far from the mean values for studies).

The estimated level of DON in the absence of visual symptoms of Fusarium head blight (the intercepts of the regression lines; $a$ ) varied among the different groups of studies, ranging from 1.8 to $3.8 \mathrm{ppm}$ (Fig. 3A). Expected DON at 0\% IND was estimated fairly precisely in studies grouped according to study type and wheat type. The width of the $95 \%$ confidence intervals around $\bar{a}$ was similar for all groupings except the Canadian location, which had a wider interval due to the smaller number of studies. The narrow interval for the entire data set was attributable to the large number of studies $(k=126)$. The estimated intercept across all studies, as well as for most groupings of studies, exceeded the 1 ppm U.S. Food and Drug Administration (FDA) advisory level for DON in wheat products destined for human consumption. The $\bar{a}$ value for all groupings were $<5 \mathrm{ppm}$, the FDA advisory level for DON in grain and grain by-products destined for animals other than cattle and chicken. The estimated IND value where $\mathrm{DON}=5 \mathrm{ppm}\left(\mathrm{IND}_{5}\right)$, and corresponding $95 \%$ confidence ("fiducial") interval was calculated using inverse-regression methods (17) (Fig. 3B). Across all studies, IND $_{5}$ was $10 \%$, with a $95 \%$ confidence interval between 6 and $16 \%$. For groupings of studies, $\mathrm{IND}_{5}$ ranged from 6 to $15 \%$, and the width of the $95 \%$ interval around $\mathrm{IND}_{5}$ was similar for all groupings, except the Canadian location and fungicide trials. The widest interval was for the Canadian location.

Model appraisal. Although there was some degree of skewness to the bivariate frequency distribution of slopes and intercepts (Fig. 1C), this histogram was not used directly to evaluate the nor- mality assumption of the random effects or the general appropriateness of the model (equation 2). The nonconstant withinstudy variability interferes with the overall evaluation. More importantly, skewness can be due to the effects of moderator variables on the effect size vector. Thus, normal quantile-quantile plots of studentized residuals, plots of studentized residuals versus predicted values, and histograms of studentized residuals were examined to evaluate appropriateness of the bivariate mixed model (17). The studentized residual is a standardization based on the within- and between-study variances.

When a model with the continuous and categorical moderator variables was fitted to the data, there was a random pattern when studentized residuals were plotted versus predicted values, and there was a symmetrical histogram of studentized residuals (L. V. Madden, unpublished data). Furthermore, there was no curvature to the normal quantile-quantile plot of studentized residuals, indicating that normality was a reasonable assumption. A few outliers were also identified with this plot. Three of four positive outliers were characterized by very large slopes. Removal of the outliers did not affect mixed model results.

\section{DISCUSSION}

The relationship between Fusarium head blight intensity and DON content in harvested wheat grain has been the subject of discussion among Fusarium head blight researchers for years. Results from individual studies have led researchers to conclusions ranging from a total lack of significant association to very strong positive relationships between Fusarium head blight and DON $(3,6,23,33,37)$. Disproportionate and, occasionally, negative relationships between DON and disease have been reported. In some instances, fairly elevated levels of DON occurred in the absence of visible symptoms of Fusarium head blight (3) or disproportionately low levels of DON occurred when the levels of visual symptoms were high $(3,37)$. However, a quantitative synthesis of the finding from 163 individual studies of association between DON and disease intensity (incidence, diseased head severity, or IND) previously showed that overall there was a significant, positive relationship (based on Pearson's product moment correlation coefficient) between DON and all commonly used field measures of Fusarium head blight intensity (31), with the strongest correlation between DON and IND.

Either the Pearson product-moment correlation or the slope (and its standard error) of a simple linear regression equation can be used to determine if there is a significant relationship between two variables (17). For a meta-analysis of a population of studies, Hunter and Schmidt (16) argue in favor of use of correlation over

TABLE 4. Influence of moderator variables at the study level on the between-study variance from bivariate random-effects meta-analysis of regression slopes and intercepts for relationship between Fusarium head blight index (IND) and deoxynivalenol (DON, ppm) content of harvested wheat grain

\begin{tabular}{|c|c|c|c|c|c|c|}
\hline \multirow[b]{3}{*}{ Moderator $^{\mathrm{a}}$} & \multicolumn{6}{|c|}{ Effect size $^{\mathrm{b}}$} \\
\hline & \multicolumn{3}{|c|}{ Slope $(b)$} & \multicolumn{3}{|c|}{ Intercept $(a)$} \\
\hline & $\hat{\sigma}_{b}^{2}$ & Difference & Reduction & $\hat{\sigma}_{a}^{2}$ & Difference & Reduction \\
\hline Location & 0.029 & 0.002 & 6.45 & 13.25 & 0.833 & 5.92 \\
\hline Wheat type & 0.029 & 0.002 & 6.45 & 13.42 & 0.664 & 4.72 \\
\hline $\bar{X}_{\mathrm{IND}}$ & 0.031 & 0.000 & 0.00 & 13.57 & 0.511 & 3.63 \\
\hline $\bar{X}_{\mathrm{DON}}$ & 0.020 & 0.011 & 35.50 & 5.42 & 8.662 & 61.50 \\
\hline$R n g_{\mathrm{IND}}$ & 0.031 & 0.000 & 0.00 & 13.95 & 0.132 & 0.94 \\
\hline$R n g_{\mathrm{DON}}$ & 0.017 & 0.014 & 45.20 & 14.01 & 0.071 & 0.50 \\
\hline
\end{tabular}

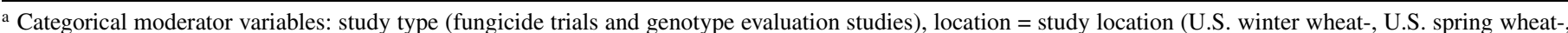
and Canadian wheat-growing regions), and wheat type (spring and winter wheat). Continuous moderator variables: $\bar{X}=$ arithmetic mean, $M a x=$ maximum, and $R n g=$ difference between maximum and mean (half-range) for IND (mean percentage of diseased spikelets per spike) and DON.

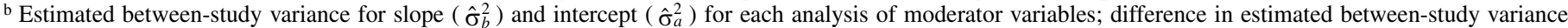
from analysis without moderator variables (Table 1) and with moderator variables, and percent reduction in between-study variances when each moderator variable was used. 
slope whenever the studies vary considerably in experimental methodology or in the nature of the measured variables (common circumstances in the social sciences, the disciplines primarily of interest to these authors). Because of the consistent methodology used and variables measured in the studies considered in our investigation of Fusarium head blight, both statistics are valid effect sizes for determining significant relationships. One advantage of the correlation coefficient is that all the information from individual studies necessary for a later meta-analysis (point estimate and sample size) is typically given in published papers. However, for determining the functional relationship between two variables, which was the goal of our current work, at least two statistics (estimated parameters, the effect sizes) must be used simultaneously for each study, such as the slope and intercept of a straight-line equation. Simultaneous analysis of two effect sizes per study requires bivariate statistical methods in order to take account of the correlation of values $(2,41)$. Until recently, bivariate (or multivariate) random-effects or mixed-effects meta-analyses were im-
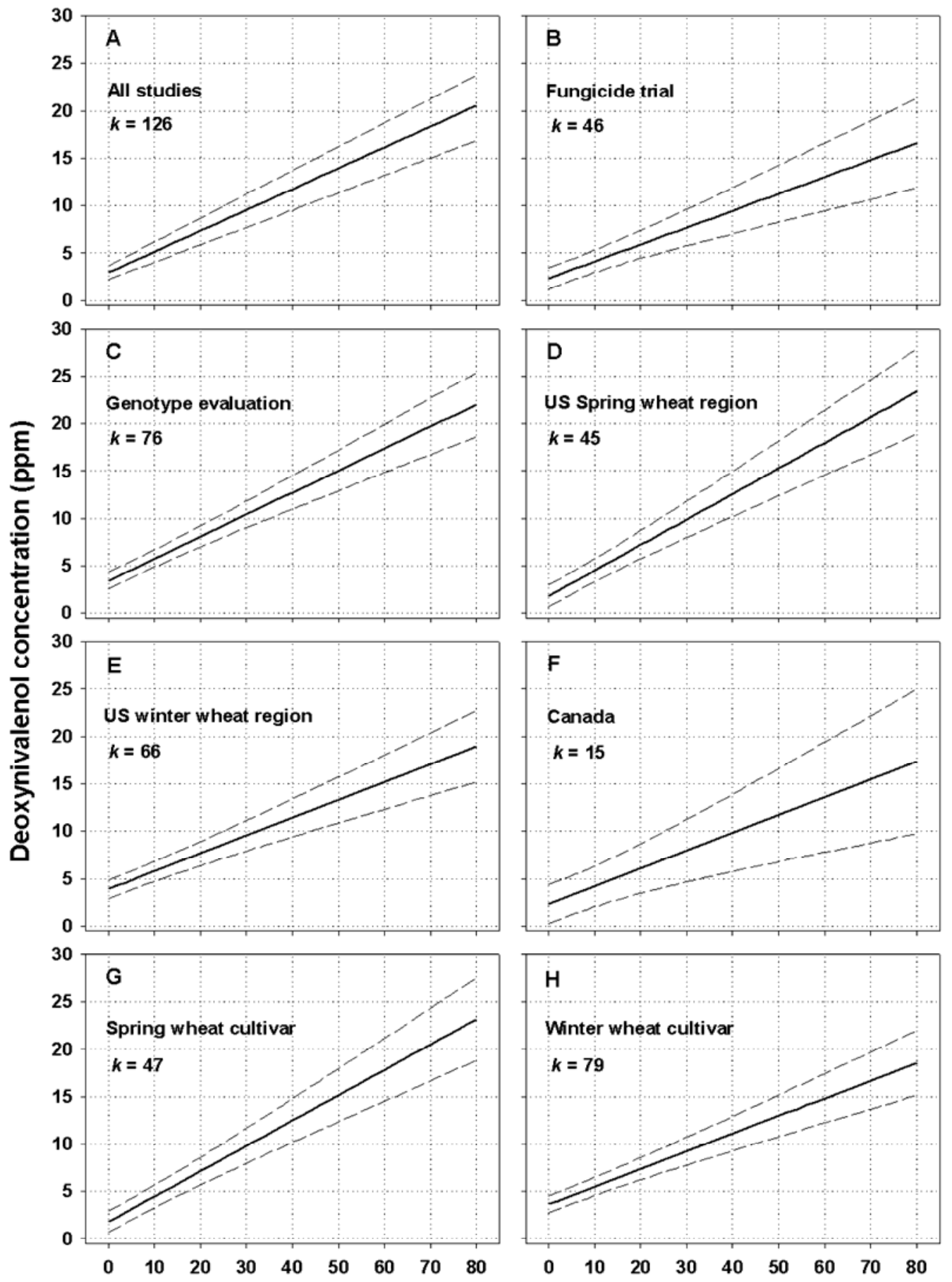

Fusarium head blight index

Fig. 2. Regression lines and their 95\% confidence intervals for relationship between Fusarium head blight index (IND; mean percentage of diseased spikelets per spike) and deoxynivalenol (DON; ppm) accumulation in harvested wheat grain. Predicted value is the estimated expected mean DON value at a given index. A, Lines and confidence intervals are shown for results from all studies used in the analysis; and groups of studies separated on the bases of B and C, study type; D, $\mathbf{E}$, and $\mathbf{F}$, study location; $\mathbf{G}$ and $\mathbf{H}$, and wheat type. $k$ is the total number of studies and the number of studies in each group. Calculations are based on the estimates of $a$ and $b$ and their standard errors in Table 2, and the estimated covariance of $a$ and $b$ determined from the linear mixed model fitted to the data. 
practical or infeasible for most investigators, mostly because of methodological challenges in model fitting and because of lack of general-purpose software. Advances in linear mixed-model theory and model fitting (34), and development of programs such as PROC MIXED of SAS have made random- and mixed-effects bivariate analyses feasible and practical for researchers (40). The required coding for meta-analysis with PROC MIXED is somewhat specialized, so van Houwelingen et al. (40) should be consulted for a thorough explanation of how to obtain appropriate results.

Through a bivariate random-effects meta-analysis of the slopes and intercepts from 126 studies, we established that there was a significant, positive straight-line relationship between DON at harvest and IND quantified at approximately growth stage 11.2. We found that the overall mean regression slope and intercept were both statistically different from zero. Based on these estimated parameters, there was on average a $0.22 \mathrm{ppm}$ increase in DON for every percent increase in IND (mean slope), and the estimated mean level of DON in the absence of
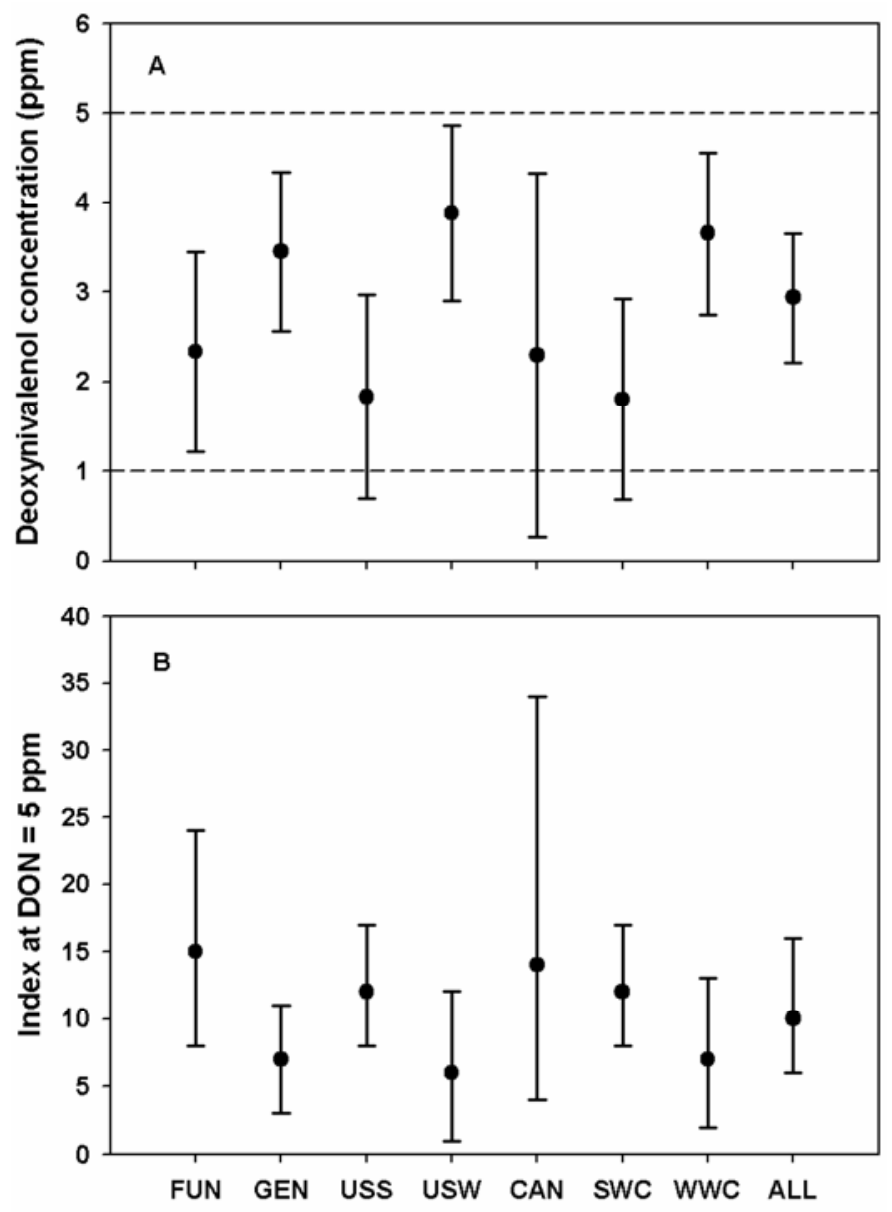

Fig. 3. Regression intercepts (estimated deoxynivalenol [DON] levels in the absence of visual symptoms of Fusarium head blight) and their 95\% confidence intervals (A) and estimated Fusarium head blight index (IND) values corresponding to an estimated DON level of $5 \mathrm{ppm}$ and their $95 \%$ confidence intervals (B) estimated from bivariate meta-analyses of regression coefficients for relationship between IND (mean percentage of diseased spikelets per spike) and DON in harvested wheat grain from 126 studies (ALL) and from studies grouped according to study type (FUN = fungicide trials and GEN = genotype evaluation studies), study location (USS = U.S. spring wheat-growing region, USW = U.S. winter wheat-growing areas, and CAN = Canadian wheat-growing areas), and wheat type ( $\mathrm{SWC}=$ spring wheat cultivars and $\mathrm{WWC}=$ winter wheat cultivars). In $\mathbf{A}$, the upper reference line represents U.S. Food and Drug Administration (FDA) advisory level for DON in grain and grain by-products destined for animals other than cattle and chicken, whereas the lower reference line represents FDA advisory level for DON in wheat products destined for human consumption. visual symptoms of Fusarium head blight (mean intercept) was $2.94 \mathrm{ppm}$.

While it is clear from this and our previous study (31) that there exists a significant relationship between IND and DON, one of the main concerns with the use of Fusarium head blight intensity as a measure of DON accumulation is the fact that DON may accumulate even in the absence of visual symptoms of Fusarium head blight (or at very low IND values), and that variation around the predicted DON can be high at any given IND value. Reports from several individual studies have alluded to this fact $(3,18,23)$. The results from this meta-analysis serve to corroborate those reports in a quantitative manner. In particular, significant betweenstudy variances (Table 1) demonstrate that studies vary considerably in the relationship between DON and IND, with a substantial range of possible DON values at any given IND value. Moreover, mean DON levels may be predicted to exceed certain threshold values even when no visual symptoms of Fusarium head blight are present. The estimated expected DON concentration at zero disease index $(\bar{a})$ represents the overall intercept value across 126 studies from multiple years and locations and not just one individual study. The overall $95 \%$ confidence interval for $\bar{a}(2.2$ to 3.8) (Fig. 3) suggests that the presence of DON in harvested grain from spikes with no visual symptoms is not just a random occurrence in an individual study, but an expected outcome when ample inoculum is present (as in these studies), irrespective of study type, wheat-growing region, and wheat type. Knowledge of moderator variables can, of course, modify this estimate and the confidence intervals, but the $95 \%$ confidence intervals exceeded 0 ppm DON in all cases.

One of the possible reasons for the accumulation of DON in the absence of visible symptoms of Fusarium head blight is the occurrence of symptomless infections. Fernando et al. (8) found that seed infection in inoculated plots was usually higher than visible disease severity and suggested that this was because of asymptomatic infection, although sampling errors could have also contributed to this difference. They speculated that lesion development may be influenced by the physiological age of the spike at the time of infection, with no symptom being expressed following late infections. Schaafsma et al. (33) used a similar argument to justify the weak correlation observed between DON accumulation and the number of viable propagules of $F$. graminearum collected with spore samplers at anthesis. They hypothesized that multiple infections and the colonization of spikes after anthesis by the fungus led to the accumulation of DON in apparently disease-free kernels. Secondary infections may be of little significance in the epidemiology of this disease (35) or in the development of visual symptoms; however, limited secondary infection could contribute to an increase in fungal biomass, and consequently, DON accumulation, without the presence of symptoms or with very limited symptom expression. The biomass of trichothecene-producing fungi is positively related to DON content of harvested wheat grain $(7,26,43)$. Lacey et al. (18) demonstrated that wheat spikes inoculated with $F$. culmorum at the late milk (Feekes growth stage 11.1)-early dough (Feekes growth stage 11.2) plant growth stage (close to the time visual assessments are commonly made) produced small amounts of DON in the absence of visual symptoms. DON-producing fungi were isolated from, and persisted in, symptomless grain throughout storage.

A large portion of the between-study variability in regression slope $\left(\hat{\sigma}_{b}^{2}\right)$ and intercept $\left(\hat{\sigma}_{a}^{2}\right)$ remain unexplained, even after adjusting for study-specific factors. Hence, the use of IND as the sole indicator of DON content in harvested grain for an individual field (in contrast to mean DON across all fields with a given IND) is questionable. DON is obviously not only a function of visible disease severity. The following factors (both unmeasured and unmeasurable) may influence this relationship: weather conditions favorable for the growth of Fusarium head blight-causing fungi and DON accumulation during the period between disease assess- 
ment and DON measurement; development of Fusarium head blight symptoms not proportional to fungal biomass; cultivar resistance to Fusarium head blight and DON accumulation; mode of action of applied fungicide; and the differential rates of DON production by $F$. graminearum isolates. These factors, individually or together, likely were responsible for the nonzero betweenstudy variance found for both slope and intercept, but their effect could not be determined directly or formally in this investigation based on the available data or the information given regarding the studies. For example, the slope or intercept could be affected if the degree of resistance (or type of resistance) differentially affects the observed IND and DON values. To directly quantify the resistance effect in a meta-analysis would require entire individual studies (and not just individual genotypes within studies) that could be categorized into different levels of resistance. However, there were no disease-screening nursery studies that could be classified as resistant or as representing any single type of resistance, only studies consisting of a collection of genotypes encompassing a range of susceptibilities. Likewise, because the environment was modified in many studies by the use of irrigation or misting, direct determination of environmental effect on the DON-IND relationship would require within-canopy measurements of weather. However, within-canopy weather measurements were not available for the majority of studies.

Our previous study (31) showed that degree of correlation between DON and IND (and other measures of Fusarium head blight intensity) was affected by categorical and continuous moderator variables. Here we showed that the functional relationship (summarized by the two effect sizes, $\bar{a}$ and $\bar{b}$ ) was also significantly affected by moderator variables. The influence of moderator variables, especially wheat-growing region, wheat type, and study-wide DON and IND levels, on the regression slope and intercept was probably due to a combination of studyspecific weather-, host-, and pathogen-related factors. For instance, studies conducted in Canada and in U.S. winter wheat areas had equivalent slopes, but significantly different intercepts. This is an indication of height differences in the regression lines and reflects differences in the levels of DON for a given level of IND between the two groups of studies, with mean DON $1.58 \mathrm{ppm}$ $(=3.88-2.30)$ higher for U.S. winter wheat than for Canadian wheat at any IND value. Differences in cultivar susceptibility to DON accumulation and differences in weather conditions between studies conducted in Canada and those conducted in U.S. winter wheat areas are likely responsible for the results. Miller et al. (26) observed that the ergosterol (an indicator of fungal biomass) to DON ratios were higher in resistant wheat cultivars than in susceptible cultivars. Thus, for any given level of fungal colonization, DON accumulation was higher in susceptible cultivars than in resistant cultivars (26). They speculated that resistant cultivars either inhibited DON synthesis or promoted DON degradation. Differences in the levels of DON between the two groups of studies in this meta-analysis also may have been due to broad differences in the DON-producing capacity and aggressiveness of isolates of $F$. graminearum from the two regions. Miller et al. (26) reported that isolates of $F$. graminearum from the United States and Canada differed in their ability to produce DON.

Although the strength of the correlation between DON and IND was clearly higher for the fungicide trials than for the genotype nursery trials (31), which was expected given the heterogeneity of susceptibilities in the latter type of study, here we found just a borderline effect of study type $(P \approx 0.06)$ on the joint effect-size vector of regression coefficients. Moreover, we found a tendency for higher slope and intercept in the genotype nursery studies than in the fungicide studies, although study type did not have a significant effect on either slope or intercept, individually (Table 2; $P>0.10)$. A larger intercept and slope could occur when some of the genotypes tested within studies have larger DON concentrations at given IND values.

Most mean slopes ranged from 0.18 to 0.23 ppm per unit IND. The only exception being for spring wheat studies (either studies conducted using spring wheat cultivars or studies conducted in traditional spring wheat areas), which had higher mean slopes (0.27 ppm per unit IND). With the exception of study type, mean intercepts were generally different among levels within each category of moderator variable, reflecting differences in DON accumulation among studies for a similar level of IND. The higher rate of change in DON with changing IND (slope) and generally higher mean levels of DON in spring than in winter wheat studies were consistent with more favorable temperature conditions in spring wheat than in winter wheat areas. This is because heading is generally in the spring for winter-wheat areas but in the summer for spring-wheat areas. Accounting for wheat growing region and other categorical moderator variables in the model, however, had only marginal effects on the estimated between-study variances, likely because of the variation in conditions (environmental and biological) within each category.

Results from the analysis of continuous moderator variables did provide further, although indirect, evidence that at least a portion of the variability in the relationship between DON and IND was due to variation in environmental factors (based on significance of the test statistics and the percent reduction in between-study variance estimates) (Tables 3 and 4). Other investigations have shown the influence of weather factors such as rainfall and temperature on either Fusarium head blight development or DON production $(15,30)$, but the influence of these specific factors (or their interactions) on the rate of change in DON with changing IND (regression slope) is unknown. When the study-wide magnitude of DON ( $\bar{X}_{\text {DON }}$ or $M a x_{\text {DON }}$ ) was used as a continuous moderator variable (and indirect indicator of conditions favorable for DON accumulation), the between-study variability in intercept was reduced by as much as $61 \%$ and the between-study variability in slope was reduced by as much as $45 \%$. In contrast, although significant, the use of IND variables in the model had less effect on the between-study variances. In general, the height of the DON:IND line (determined by $a$ ) increased with increasing $\bar{X}_{\text {IND }}$ and $\bar{X}_{\mathrm{DON}}$ (as well as other measures of DON), suggesting that the mean DON for a given IND value increases as overall conditions become more favorable for disease development and toxin production. Moreover, the rate of change of DON per unit change in IND $(b)$ increased with increasing DON levels of studies. Although of value in understanding the DON-IND relationship, the effects of DON on the relationship obviously are not directly useful for improving predictions, since overall DON level at harvest is not known when disease is assessed at growth stage 11.2; that is, one would require some of the information that is being predicted in order to improve predictions.

Part of the unexplained variability in the relationship between DON and IND may have been due to errors made during disease assessment and DON quantification; in particular, performing DON analysis on a sample of grain that was not representative of the lot being sampled. Standard determination of DON at harvest is based on a bulked sample of a small quantity of ground-up seeds $(10,12)$, likely originating from spikes with different levels of disease. Only some of the seeds from diseased spikes would be infected in the bulked sample, unless incidence and severity were both $100 \%$ in the sample. Thus, measured DON in an assay typically is a composite value for seeds with different levels of DON (including those with $0 \mathrm{ppm}$ ) and different levels of disease. Since the spatial distribution of infected and DON-contaminated seeds in a lot may vary with disease intensity, Hart and Schabenberger (12) proposed the use of an adaptive sampling protocol for DON quantification, based on disease intensity. Based on their results, the commonly used systematic, single probe sampling protocol may provide an unbiased estimate of DON in 
an epidemic year when the distribution of contaminated seeds within a lot is fairly homogeneous. However, in nonepidemic years, when the distribution on contaminated seeds is likely to be less homogeneous, such a protocol may lead to biased estimates of DON levels. Hence, it may be of interest to alter sampling intensity based on disease level in order to achieve more precise and reliable DON estimates.

In conclusion, through this meta-analysis, we have established that there is a significant linear relationship between Fusarium head blight disease index and DON accumulation in wheat grain. However, given the magnitude of the between-study variances and the fact that DON may accumulate in the absence of visual symptoms (or at very low disease index values), caution should be exercised when using IND as the sole predictor of individual DON values. The results with the moderator variables suggest that the relationship may be affected by genetic and environmental factors. Additional research is needed to determine if and how the slope and intercept are affected by increasing host resistance within a single wheat type (e.g., soft red winter), type of resistance (2), and environmental conditions measured within the wheat canopy. Related to this, specific effects of tillage practices on the relationship should be investigated, although we predict that, although tillage may affect the magnitude of IND and DON $(6,33)$, it will have little effect on the relationship between the two. Once additional information is available, it should be possible to determine through meta-analysis if earlier predictions of DON accumulation in wheat grain can be made based on IND and other moderator variables.

\section{ACKNOWLEDGMENTS}

Salaries and research support were provided by state and federal funds, especially the U.S. Wheat and Barley Scab Initiative, to the Ohio Agricultural Research and Development Center, The Ohio State University. We thank D. Hershman, C. Sneller, G. Milus, and M. McMullen for providing some of the data utilized in this study.

\section{LITERATURE CITED}

1. Bai, G., and Shaner, G. 1994. Scab of wheat: Prospects for control. Plant Dis. 78:760-766.

2. Berkey, C. S., Hoaglin, D. C., Antczak-Bouckoms, A., Mosteller, F., and Colditz, G. A. 1998. Meta-analysis of multiple outcomes by regression with random effects. Stat. Med. 17:2537-2550.

3. Champeil, A., Fourbet, J. F., Dore, T., and Rossignol, L. 2004. Influence of cropping system on Fusarium head blight and mycotoxin levels in winter wheat. Crop Prot. 23:531-537.

4. DeWolf, E. D., Madden, L. V., and Lipps, P. E. 2003. Risk assessment models for wheat Fusarium head blight epidemics based on within-season weather data. Phytopathology 93:428-435.

5. DeWolf, E., Lipps, P., Miller, D., Knight, P., Molineros, J., Francl, L., and Madden, L. 2004. Evaluation of prediction models for wheat Fusarium head blight in the U.S., 2004. Page 439 in: Proc. 2nd Int. Symp. Fusarium Head Blight. Michigan State University, East Lansing.

6. Dill-Macky, R., and Jones, R. K. 2000. The effect of previous crop residues and tillage on Fusarium head blight of wheat. Plant Dis. 84:7176.

7. Edwards, S. G., Pirgozliev, S. R., Hare, M. C., and Jenkinson, P. 2001. Quantification of trichothecene-producing Fusarium Species in harvested grain by competitive PCR to determine efficacies of fungicides against Fusarium head blight of winter wheat. Appl. Environ. Microbiol. 67: $1575-1580$

8. Fernando, W. G. D., Paulitz, T. C., Seaman, W. L., Dutilleul, P., and Miller, J. D. 1997. Head blight gradient caused by Gibberella zeae from area sources of inoculum in wheat field plots. Phytopathology 87:414421.

9. Field, A. P. 2001. Meta-analysis of correlation coefficients: A Monte Carlo comparison of fixed- and random-effects methods. Psychol. Methods 6:161-180.

10. Hart, L. P., Casper, H., Schabenberger, O., and Ng, P. 1998. Comparison of gas chromatography and enzyme linked immunosorbent assay for deoxynivalenol in milled fractions of naturally contaminated wheat. J.
Food Prot. 61:1695-1697.

11. Hart, L. P., Pestka, J. J., and Liu, M. T. 1984. Effect of kernel development and wet periods on production of deoxynivalenol in wheat infected with Gibberella zeae. Phytopathology 74:1415-1418.

12. Hart, L. P., and Schabenberger, O. 1998. Variability of vomitoxin in truckloads of wheat in a wheat scab epidemic year. Plant Dis. 82:625-630.

13. Hedges, L. V., and Vevea, J. L. 1998. Fixed- and random-effects models in meta-analysis. Psychol. Methods 3:486-504.

14. Hooker, D. C., Schaafsma, A. W., and Tamburic-Ilincic, L. 2002. Using weather variables pre- and post-heading to predict deoxynivalenol content in winter wheat. Plant Dis. 86:611-619.

15. Hope, R., Aldred, D., and Magan, N. 2005. Comparison of environmental profiles for growth and deoxynivalenol production by Fusarium culmorum and F. graminearum on wheat grain. Lett. Appl. Microbiol. 40:295-300.

16. Hunter, J. E., and Schmidt, F. L. 2004. Methods of Meta-Analysis: Correcting Error and Bias in Research Findings. 2nd ed. Sage Publications, Inc., Thousand Oaks, CA.

17. Kutner, M. H., Nachtsheim, C. J., Neter, J., and Li, W. 2005. Applied Linear Statistical Models. McGraw-Hill/Irwin, New York.

18. Lacey, J., Bateman, G. L., and Mirocha, C. J. 1999. Effects of infection time and moisture on development of ear blight and deoxynivalenol production by Fusarium spp. in wheat. Ann. Appl. Biol. 134:277-283.

19. Large, E. C. 1954. Growth stages in cereals. Plant Pathol. 3:128-129.

20. Lipps, P. E., Madden, L., Mills, D. R., and Paul, P. 2005. Forecasting Fusarium head scab of wheat in Ohio in 2005 and summary of disease levels, 2002-2005. Ohio Agricultural Research and Development Center, Plant Pathology Department Series 128.

21. Lipsey, M. W., and Wilson, D. B. 2001. Practical Meta-Analysis. Sage Publications, Inc., Thousand Oaks, CA.

22. Littell, R. C., Milliken, G. A., Stroup, W. W., and Wolfinger, R. D. 1996. SAS System for Mixed Models. SAS Institute, Cary, NC.

23. Liu, W., Langseth, W., Skinnes, H., Elen, O. N., and Sundheim, L. 1997. Comparison of visual head blight ratings, seed infection levels, and deoxynivalenol production for assessment of resistance in cereals inoculated with Fusarium culmorum. Eur. J. Plant Pathol. 103:589595.

24. Madden, L. V., Lipps, P. E., and De Wolf, E. 2004. Developing forecasting systems for Fusarium head blight. Page 471 in: Proc. 2nd Int. Symp. Fusarium Head Blight. Michigan State University, East Lansing.

25. McMullen, M., Jones, R., and Gallenburg, D. 1997. Scab of wheat and barley: A re-emerging disease of devastating impact. Plant Dis. 81:13401348.

26. Miller, J. D., Young, J. C., and Sampson, D. R. 1985. Deoxynivalenol and Fusarium head blight resistance in spring cereals. Phytopathol. Z. 113:359-367.

27. Nganje, W. E., Bangsund, D. A., Leistritz, F. L., Wilson, W. W., and Tiapo, N. M. 2004. Regional economic impact of Fusarium head blight in wheat and barley. Rev. Agric. Econ. 26:322-347.

28. Normand, S.-L. T. 1999. Tutorial in biostatistics. Meta-analysis: Formulating, evaluating, combining, and reporting. Stat. Med. 18:321-359.

29. Parry, D. W., Jenkinson, P., and McLeod, L. 1995. Fusarium ear blight (scab) in small grain cereals-A review. Plant Pathol. 44:207-238.

30. Paul, P. A., El-Allaf, S. M., Lipps, P. E., and Madden, L. V. 2004. Rain splash dispersal of Gibberella zeae within wheat canopies in Ohio. Phytopathology 94:1342-1349.

31. Paul, P. A., Lipps, P. E., and Madden, L. V. 2005. Relationship between visual estimates of Fusarium head blight intensity and deoxynivalenol accumulation in harvested grain: A meta-analysis. Phytopathology 95:1225-1236

32. Rotter, B. A., Prelusky, D. B., and Pestka, J. J. 1996. Toxicology of deoxynivalenol (vomitoxin). J. Toxicol. Environ. Health 48:1-34.

33. Schaafsma, A. W., Tamburic-Ilincic, L., and Hooker, D. C. 2005. Effect of previous crop, tillage, field size, adjacent crop, and sampling direction on airborne propagules of Gibberella zeae/Fusarium graminearum, Fusarium head blight severity, and deoxynivalenol accumulation in winter wheat. Can. J. Plant Pathol. 27:217-224.

34. Schabenberger, O., and Pierce, F. J. 2002. Contemporary Statistical Models for the Plant and Soil Sciences. CRC Press, New York.

35. Shaner, G. 2003. Epidemiology of Fusarium head blight of small grain cereals in North America. Pages 84-119 in: Fusarium Head Blight of Wheat and Barley. K. J. Leonard and W. R. Bushnell, eds. The American Phytopathological Society, St. Paul, MN.

36. Sheu, C. F., and Suzuki, S. 2001. Meta-analysis using linear mixed models. Behav. Res. Methods Instr. C. 33:102-107.

37. Snijders, C. H. A., and Perkowski, J. 1990. Effect of head blight caused by Fusarium culmorum on toxin content and weight of wheat kernels. Phytopathology 80:566-570.

38. Stack, R. W., and McMullen, M. P. 1998. A visual scale to estimate severity of Fusarium head blight in wheat. NDSU Extension Service: 
Small Grains Publications. Online Publication/PP-1095.

39. Stijnen, T. 2000. Tutorial in biostatistics. Meta-analysis: Formulating, evaluating, combining, and reporting by S.-L. T. Normand, Stat. Med. 18:321-359 (1999). Stat. Med. 19:159-161.

40. Van Houwelingen, H. C., Arends, L. R., and Stijnen, T. 2002. Advanced methods in meta-analysis: Multivariate approach and meta-regression. Stat. Med. 21:589-624.

41. van Houwelingen, H., Zwinderman, K., and Stijnen, T. 1993. A bivariate approach to meta-analysis. Stat. Med. 12:2272-2284.
42. Verbeke, G., and Lesaffre, E. 1997. The effect of misspecifying the random effects distribution in linear models for longitudinal data. Comput. Stat. Data An. 23:541-556.

43. Wang, Y. Z., and Miller, J. D. 1988. Effects of Fusarium graminearum metabolites on wheat tissue in relation to Fusarium head blight resistance. J. Phytopathol. 122:118-125.

44. Windels, C. E. 2000. Economic and social impacts of Fusarium head blight: Changing farms and rural communities in the Northern Great Plains. Phytopathology 90:17-21. 